\title{
Regulation of Lipid Metabolism by Lamin in Mutation-Related Diseases
}

\author{
Yue Peng ${ }^{1 \dagger}$, Qianyu Tang $^{1 \dagger}$, Fan $\mathrm{Xiao}^{2 *}$ and Nian $\mathrm{Fu}^{1,2 *}$ \\ ${ }^{1}$ The Affiliated Nanhua Hospital, Department of Gastroenterology, Hunan Provincial Clinical Research Center of Metabolic \\ Associated Fatty Liver Disease, Hengyang, China, ${ }^{2}$ The Affiliated Nanhua Hospital, Clinical Research Institute, Hengyang Medical \\ School, University of South China, Hengyang, China
}

OPEN ACCESS

Edited by:

Ana Blas-García,

University of Valencia, Spain

Reviewed by:

Giuseppe Novelli,

University of Rome Tor Vergata, Italy Joshi Stephen,

Baylor College of Medicine,

United States

*Correspondence:

$\mathrm{Nian} \mathrm{Fu}$

2002funian@163.com

Fan Xiao

xiaof-usc@foxmail.com

${ }^{\text {t}}$ These authors have contributed equally to this work

Specialty section:

This article was submitted to Gastrointestinal and Hepatic

Pharmacology,

a section of the journal

Frontiers in Pharmacology

Received: 23 November 2021

Accepted: 24 January 2022

Published: 25 February 2022

Citation:

Peng Y, Tang Q, Xiao F and Fu N (2022) Regulation of Lipid Metabolism

by Lamin in Mutation-

Related Diseases.

Front. Pharmacol. 13:820857.

doi: $10.3389 /$ fphar.2022.820857
Nuclear lamins, known as type 5 intermediate fibers, are composed of lamin A, lamin C, lamin B1, and lamin B2, which are encoded by LMNA and LMNB genes, respectively. Importantly, mutations in nuclear lamins not only participate in lipid disorders but also in the human diseases, such as lipodystrophy, metabolic-associated fatty liver disease, and dilated cardiomyopathy. Among those diseases, the mechanism of lamin has been widely discussed. Thereby, this review mainly focuses on the regulatory mechanism of the mutations in the lamin gene in lipid alterations and the human diseases. Considering the protean actions, targeting nuclear lamins may be a potent therapeutic avenue for lipid metabolic disorders and human diseases in the future.

Keywords: nuclear lamins, lipid metabolism, human diseases, mutation of lamin, lipodystrophy

\section{INTRODUCTION}

The nuclear lamina $(\mathrm{NL})$ is fibrin network structure located in the lower layer of nuclear membrane, which is primarily composed of lamin A, C, B1, and B2 in mammalian cells. Lamins A and C are classified as type A encoded by $L M N A$ while $\mathrm{B} 1$ and $\mathrm{B} 2$ as type $\mathrm{B}$ encoded by $L M N B 1$ and $L M N B 2$, separately (Figure 1). As a major component of the nuclear lamina, lamin is responsible for maintaining the nuclear shape, transducting signals, organizing chromatin, repairing DNA, and pyroptosis (de Leeuw et al., 2018; Stiekema et al., 2020). In structure, lamin, which serves as a V-type intermediate filaggrin (IFS), forms the main cytoskeleton of the nucleus. Like all IFS, a V-type IFS has three components, mainly including an amino acid domain at the head, a helix domain at the center, and a carboxy-terminal domain at the tail. Additionally, the unique characteristics of these subcomponents mainly include a nuclear localization signal (NLS), IG folding domain, and CaaX motif $(\mathrm{C}=$ cysteine, $\mathrm{A}=$ aliphatic residue, $\mathrm{X}=$ any residue) (Gruenbaum and Medalia, 2015; Dittmer and Misteli, 2011) (Figure 2). Surpringly, the CaaX motif is a vital post-translational modification site for lipid metabolism (Kuchay et al., 2019). Therefore, considering the above structure of lamin, lamin may be an indispensable part in maintaining lipid homeostasis.

Indeed, the structure of lamin is of great importance to lipid metabolism. The dysfunction of lamin leads to numerous pathologies, mainly affecting the structure of the nuclear membrane, lipid synthesis genes, transcription factors, degenerative pathology, fat distribution, malnutrition, and aging (Östlund et al., 2020; Kim et al., 2018; Afonso et al., 2016; Ruiz de Eguino et al., 2012; Padiath and $\mathrm{Fu}, 2010)$. So, the abnormity of lamin elicits a series of metabolic disorders, the most common of which is lipodystrophy (Maraldi et al., 2011). The correlation between lamin and lipid metabolism has been well elucidated. For instance, the overexpression (OE) of lamin B1 downregulates the expression of lipid synthesis genes and the content of myelin-enriched lipids, ultimately increasing the risk of autosomal dominant leukodystrophy (ADLD) (Östlund et al., 2020). Also, NF- $\kappa B$ is activated by lamin $\mathrm{A} / \mathrm{C}$, subsequently boosting proinflammatory genes, such as $\mathrm{Il} 6, \mathrm{Tnf}, \mathrm{Ccl} 2$, and 


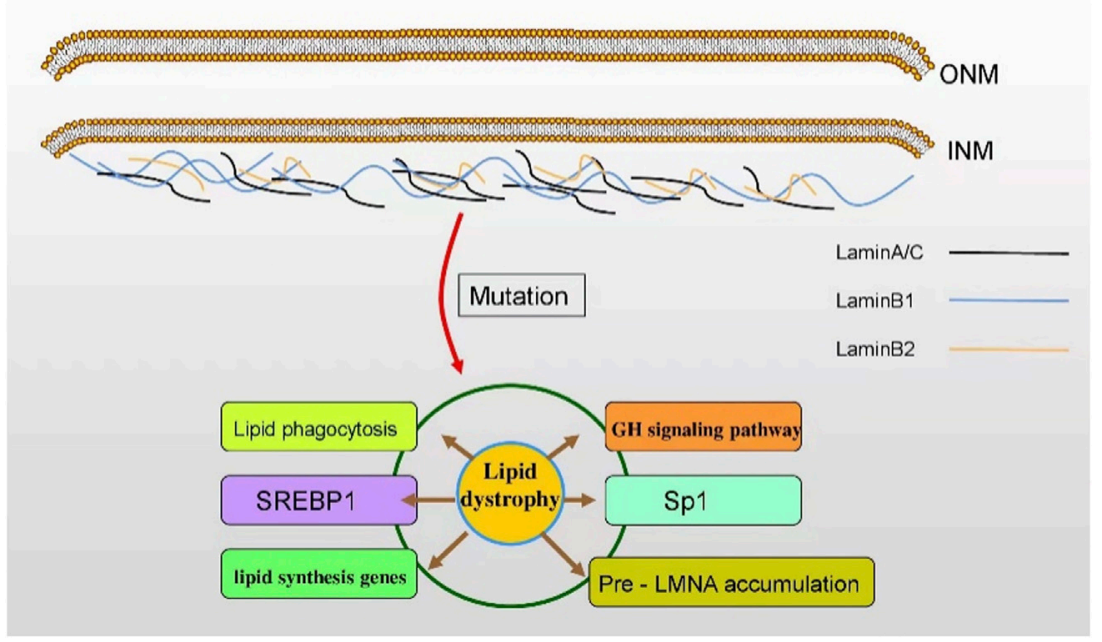

FIGURE 1 | The subtypes and roles of lamin. Lamin AVC, lamin B1, and lamin B2 are the fibrin network structures located at the lower layer of the inner nuclear membrane. Lamin is responsible for maintaining the nuclear shape, participating in signal transduction, organizing chromatin, repairing DNA, and inducing apoptosis. The mutation of LMN genes affect lipid dystrophy in various human diseases. INM: inner nuclear membrane; ONM: outer nuclear membrane.

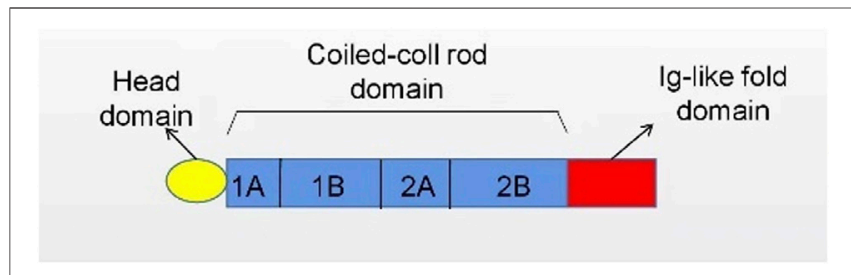

FIGURE 2 | The structure of lamin. Nuclear lamins contain three domains: a head domain, a coiled-coil rod domain, and an lg-like fold domain.

Nos2, and finally promoting the development of obesity-induced insulin resistance in adipose tissue macrophages (ATMs) (Kim et al., 2018). As lamin always interferes with lipid metabolism, it is plausible that lamin-mediated lipid disorders may be intimately correlated with human diseases. Indeed, accumulating evidence has demonstrated that the mutations in the lamin gene play an important role on human diseases. Lamin genes are susceptible to mutate, hundreds of which are correlated to the human diseases. Furthermore, it is noteworthy that $17 \%$ of those diseases are lipodystrophy. Lipodystrophy syndrome is a rare heterogeneous disease characterized by systemic or partial fat atrophy with metabolic complications, which include insulin resistance, diabetes mellitus (DM), female hyperandrogenia, fatty liver, and dyslipidemia. Then, numerous studies have shown that lamin mutations are of great importance in human lipodystrophy syndrome (Table 1). More specifically speaking, lamin $\mathrm{A} / \mathrm{C}$ mutation, prelamin $\mathrm{A}$ maturation, and lamin $\mathrm{B}$ mutation or deregulation have been proven to be the reasons or significant related factors of human lipodystrophy syndrome (Guénantin et al., 2014; Laver et al., 2018). Since lamin is associated with various lipid-related physiological alterations, more emphasis should be placed on the mechanism of the nuclear lamins in human diseases. Herein, this review summarizes the molecular mechanisms of lamin mutationassociated diseases concerning lipid metabolism.

TABLE 1 | Nuclear lamina-related diseases about genetic lipodystrophy syndromes.

\section{Lipodystrophy type}

Familial partial lipodystrophy type 2 (FPLD2)

Hutchinson-Gilford progeria syndrome (HGPS) Mandibuloacral dysplasia with lipodystrophy (MAD type A)

Mandibuloacral dysplasia with lipodystrophy

(MAD type B)

Atypical Werner syndrome (AWS)

Adult-onset demyelinating leukodystrophy (ADLD)

Acquired partial lipodystrophy (APL)

\section{Genetic mutation}

LMNA (151660 AD)

LMNA (176670 AD)

LMNA (248370 AR)

ZMPSTE24

(608612 AR)

LMNA (150330 AR)

LMNB1 (169500 AD)

LMNB2 (608709 AD)

\section{Clinical phenotype}

Gradual loss of fat from the limbs and trunk, "cushingoid" appearance due to neck and face sparing, muscular dystrophy, dilated cardiomyopathy Generalized loss of subcutaneous fat, progeroid features

Mandibular and clavicular hypoplasia, acro-osteolysis. Distal and truncal lipoatrophy, progeroid features

Mandibular and clavicular hypoplasia, acro-osteolysis. More generalized loss of fat, premature renal failure, progeroid features

Partial or generalized loss of subcutaneous fat, progeroid features

Downregulates the expression of genes associated with lipid synthesis, which in turn leads to a decrease in myelin-rich lipids

Gradual symmetrical subcutaneous fat loss, starting in the face and progressing down the upper part of the body. Subcutaneous fat in the lower abdomen and legs is significantly reduced, while fat storage in the gluteal area and lower limbs tends to be retained or increased 


\section{THE REGULATORY MECHANISMS IN HUMAN DISEASES BY MUTATION OF LAMIN GENES}

In the human disease spectrum, hundreds of mutations in the LMNA gene have been identified and are associated with more than a dozen human diseases, especially including lipodystrophy (Gonzalo et al., 2017). Lipodystrophy is a series of heterogeneous diseases characterized by the loss of selective adipose tissue or loss of functional adipose cells, thus leading to dyslipidemia and heterotopic steatosis (Hafidi et al., 2019). Specifically speaking, autosomal dominant mutations in $L M N A$ genes are strongly correlated with familial partial lipodystrophy type 2 (FPLD type 2) (Hegele et al., 2000; Jéru et al., 2017). Additionally, the autosomal recessive mutation of $L M N A$ gene is related to mandibuloacral dysplasia (MAD), which causes changes in lipid metabolism (Bagias et al., 2020). Besides, the mutation of LMN gene also elicits a dozen of diseases, including FPLD, Hutchinson-Gilford progeria syndrome (HGPS), metabolic associated fatty liver disease (MAFLD), MAD, dilated cardiomyopathy (DCM), autosomal dominant leukodystrophy (ADLD), acquired partial lipodystrophy (APL), Barraquer-Simons (BSS) syndrome, atypical Werner syndrome (WS), limb girdle muscular dystrophy type 1b (LGMD1B), and the autosomal dominant form of Emery-Dreifuss muscular dystrophy (AD-EDMD). In recent years, the regulatory mechanism of lamin mutation has been gradually elucidated. However, the understanding of the regulatory mechanism of mutated lamin in human diseases is not entirely clear, which is still being explored. The study on the regulatory mechanism of lamin mutation is helpful to reveal the importance of lamin in human diseases.

\section{LAMINA/C MUTATION-RELATED DISEASES}

\section{The Mutation of LMNA Increases Prelamin A Accumulation}

FPLD2, a large genetic and phenotypic variation first reported in the 1970s, is characterized by the progressive loss of subcutaneous adipose tissue in the limbs and trunk, accumulation of fat in the face and neck, and severe metabolic disorders, including insulin resistance, glucose intolerance, diabetes, dyslipidemia, and steatohepatitis (Dunnigan et al., 1974; Köbberling et al., 1975; Krawiec et al., 2016). Interestingly, LMNA R482W and R482Q are common pathogenic variants in FPLD type 2 (Özen et al., 2020). FPLD and HGPS both belong to premature aging diseases and exhibit a significant loss of subcutaneous adipose tissue. More intriguingly, the pathogenesis of HGPS is caused by the LMNA mutation, preventing the conversion of prelamin A to mature lamin A, thereby leading to the accumulation of prelamin A. Later, a large number of studies reported the pathogenesis of lipodystrophy in FPLD roots in the accumulation of prelamin A by mutated LMNA, which is similar to the pathogenesis of HGPS (Capanni et al., 2005; Bidault et al., 2013; Afonso et al., 2016).
Conversely, Tu et al. (2016) doubted that most of the mutated sites of LMNA mutation in FPLD are not located in the key sequence for lamin A processing. In addition to this, some of these commercial antibodies bind nonspecifically to other proteins. To reconfirm the mechanisms involved, the monoclonal antibodies against prelamin $\mathrm{A}$ made by $\mathrm{Tu}$ were used for four subjects with LMNA mutations in lipodystrophy. Surprisingly, no evidence of prelamin A accumulation was found. As a result, $\mathrm{Tu}$ et al. suggested that the missense mutations of LMNA in FPLD cannot lead to an accumulation of prelamin A.

Meanwhile, a growing number of studies have shown that the function of lamin not only at the cellular level but also in disease states is controlled by the PTMs of proteins, including phosphorylation (Machowska et al., 2015), SUMOylation (Moriuchi et al., 2016), glycosylation (Snider and Omary, 2014), farnesylation (Farnsworth et al., 1989), methylation (Rao et al., 2019), o-glcNAcylation (Alfaro et al., 2012; Wang et al., 2012; Simon et al., 2018), succinylation (Weinert et al., 2013), and ubiquitination (Wagner et al., 2011; Povlsen et al., 2012). Farnesylation is special among many PTMs in the regulation of prelamin A. Specifically speaking, pre- $L M N A$ (the precursor of mature LMNA) and B-type LMN are a fannification on the cysteine residues of carboxy-terminalCaax motifs (Weber et al., 1989). Moreover, the three terminal amino acids on type A and type B LMN were subjected to zinc metalloproteinases (ZMPSTE24; prelamin A) or RAS-converting enzyme 1 (Rce1; LMNB1 and B2) and cleaved by methylated $\alpha$ -carboxyl groups (Winter-Vann and Casey, 2005). Interestingly, $L M N C$ is not able to be fannized due to lack of the -Caax motif (Goldberg et al., 2008; Adam et al., 2013; Jung et al., 2013). Therefore, ZMPSTE24 is essential for prelamin A to become a mature lamin A. Afonso et al. (2016) discovered that the expression of ZMPSTE24 decreases in FPLD cells, but it is not clear why $L M N A / C$ missense mutation affected the expression of ZMPSTE24. Therefore, for lipid dystrophy caused by LMNA mutation in FPLD, does LMNA mutation cause prelamin A accumulation? If there is an accumulation of prelamin $\mathrm{A}$, is it caused by $L M N A$ mutation that reduces the expression of ZMPSTE24?

In short, there is much evidence that the accumulation of prelamin A by mutated LMNA causes FPLD and HGPS. Since prelamin $\mathrm{A}$ has a paradoxical role in the occurrence and development of FPLD and HGPS, the relevant regulatory mechanism of $L M N A$ mutation remains to be further explored.

\section{The Mutated Lamin Elicits Dynamic Recombination of Nuclear Layer Networks}

The rupture of lamina is an early event and a prerequisite of lipogenesis and lipocyte differentiation. To understand the underlying regulatory networks, Verstraeten et al. (2011) have found broken lamina, the loss of lamin, and emerin proteins at the 10th day of fat cell differentiation. Eight days after that, the proportion of cells expressing lamins increase while lamin A/C protein levels remain low in the whole. Thus, the re-expression of lamin subtypes increases the plasticity of nuclear membrane to indentations under lipid stress, ultimately causing a reorganization 
of the cellular infrastructure. Moreover, progerin, a farnylated protein resulting from $L M N A$ mutation, can harden the nucleus and reduce the reorganization, finally wiping the differentiation of lipocytes. Lipid accumulation happens, while low progerin expresses (Najdi et al., 2021). In short, targeting progerin may be an effective method to improve lipid disorders.

\section{The Mutation of LMNA Enhances Lipophagy}

Lipophagy is mainly manifested as the interaction of the autophagosome membrane with LC3. Then, the lipid droplets (LDs) are selectively delivered to the lysis chamber for degradation by the autophagic protein (Singh and Cuervo, 2012; Wang, 2016; Kloska et al., 2020; Shin, 2020). In a recent study, Chad A. Cowan et al. (Friesen and Cowan, 2018) discovered that in lipolysis, the proportion of LC3-II and LC3I, the level of ATG7 protein significantly stimulates LMNA R482W mutant cells, thereby indicating that autophagosome formation increases in FPLD2 adipocytes. As described above, reduced fat production, increased lipolysis, and increased autophagy may be intimately related to the lipid abnormalities of FPLD2. As a result, LMNA mutation promotes lipophagy, while the deeper connection between the lamin gene and lipophagy needs more exploration.

\section{The Lamin A/C Activates Liver Growth Hormone Receptor Signals}

MAFLD, a clinicopathological syndrome, is characterized by an excessive deposition of fat in liver cells caused by non-alcohol and other clear liver damage factors and is closely related to metabolic stress liver injury (Mantovani and Dalbeni, 2020). Recently, Vargas et al. (Mahdi et al., 2020) indicated that a case of MAFLD patient was derived from the D300N LMNA mutation of FPLD, which surprisingly suggested that the mutation of the lamin gene may progress to steatosis, therefore eliciting MAFLD. Accordingly, the subsequent genetic testing and the risk of MAFLD should be taken seriously in FPLD-diagnosed patients. Beyond that, the change of lamin protein may also contribute to the occurrence and development of MAFLD. Recent research reported that the specific lamin A/C deficiency of hepatocellular in mice induces spontaneous liver injury and increases the susceptibility to steatohepatitis fed with high-fat diets in mice (Kwan et al., 2017). Considering the fact mentioned above, it is plausible that lamin protein is intimately correlated with MAFLD.

Indeed, the regulatory mechanism of lamin in MAFLD has been gradually elucidated. Nevertheless, the understanding of the regulatory mechanism remains unclear. It has been found that the deficiency of lamin A/C upregulates stat1 mRNA and protein levels and blocks the phosphorylation of Janus kinase 2 (JAK2), transcription activator (Stat 5) and extracellular regulated protein kinases (ERKs) mediated by the liver growth hormone (GH) receptor signal, thus downregulating the expression of stat5dependent male-specific genes, ultimately promoting excessive fatty acids, inflammation, and fibrosis in hepatocytes and exacerbating the progression of MAFLD (Kwan et al., 2017) (Figure 3).
In general, the mutation of the LMNA gene induces MAFLD. Similarly, the GH signal pathway and stat1 mediated by lamin $\mathrm{A} / \mathrm{C}$ play protective roles in delaying MAFLD progression. Nevertheless, the regulatory mechanism of lamin in MAFLD remains to be further studied.

\section{Prelamin A Segregates SREBP1 at Nuclear Margin}

$\mathrm{MAD}$, an extremely rare autosomal recessive disorder, is mainly manifested as bone abnormalities, premature aging, and lipodystrophy (Bagias et al., 2020). Growing evidence suggests that the lamin mutation may be a vital part in the lipid changes of MAD. Firstly, a study hinted that a single amino acid substitution in laminin A/C causes MAD (Novelli et al., 2002). In addition, the two defects in MAD are the mutation of LMNA or ZMPSTE24 genes and are named as type $\mathrm{A}$ or $\mathrm{B} \mathrm{MAD}$, respectively. Intriguingly, it has been found that the different types of MAD also cause various fat changes. Specifically speaking, type A MAD (MADA) loses fat in the extremities, while fat deposits in the neck and trunk are normal or excessive; type $B$ MAD (MADB) forms subcutaneous fat loss that resulted from a typical mutation in the ZMPSTE24 gene (Cenni et al., 2018). The above evidence shows that the mutated lamin is intimately correlated with lipid disorders in MAD. Moreover, some studies have demonstrated that the regulatory mechanism of prelamin A plays an important role in MAD.

Indeed, some studies have demonstrated that prelamin A plays an important role in regulating lipid homeostasis. Lattanzi et al. (Capanni et al., 2005) reported that prelamin A accumulated in Dunnigan familial partial lipodystrophy, mandibuloacral dysplasia, and atypical Werner syndrome, which are three laminopathies characterized by lipodystrophic phenotypes. Furthermore, prelamin A precursors specifically accumulate in dystrophic cells and lipodystrophic cells and colocalize with cholesterol regulatory element binding protein 1 (SREBP1) (SREBP1: The key regulators of lipid metabolism, involved in adipocyte differentiation, are expressed at high levels in adipose tissue, and stimulates the expression of a variety of adipogenic genes, including FAS, acetyl-CoA carboxylase, stearoyl-coenzyme A desaturase1, and lipoprotein lipase ( $\mathrm{Li}$ et al., 2016). Lattanzi et al. also suggested that not mature lamin A/C but rather prelamin A interacts with SREBP1. The mechanism is that prelamin A isolates SREBP1 at the nuclear border, thereby declining the activation of peroxisome proliferator activated receptor gamma (PPAR gamma) via inhibiting active SREBP1, thus impairing preadipocyte differentiation (Capanni et al., 2005).

In conclusion, the lamin mutation leads to lipid changes in MAD. Also, prelamin A interacts with SREBP1, thus affecting the actions of pre-adipocytes. However, the understanding of the lipid-regulated mechanism of lamin is not entirely clear, which is still being explored.

\section{Prelamin A and Sp1 Effects on Adipogenesis}

To investigate the effect of lamin on transcriptional factors, research shows that lipodystrophy can not only result from 


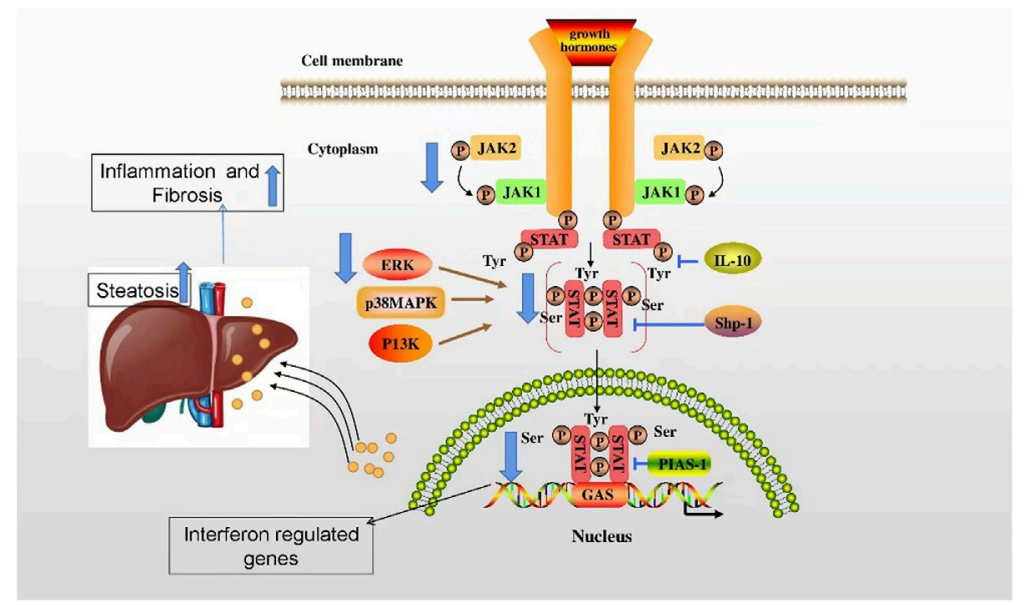

FIGURE 3 | The regulatory mechanism of lamin in alleviation of MAFLD. The deficiency of lamin A/C upregulates stat1 mRNA and protein levels and blocks JAK2, Stat 5, and ERK mediated by the liver GH receptor signal, thus downregulating the expression of stat5-dependent male-specific genes, ultimately promoting excessive fatty acids, inflammation, and fibrosis in hepatocytes and exacerbating the progression of MAFLD.

lamin mutation but also from the use of human immunodeficiency virus protease inhibitors (PIs). Under PI treatment in human mesenchymal stem cells (hMSC), impaired adipogenesis is due to an interaction between accumulated prelamin A and Sp1 transcription factors, finally altering extracellular matrix gene expression (Ruiz de Eguino et al., 2012). In general, the interaction between prelamin A and Sp1 excacerbates $L M N A$-linked lipostrophy. Nevertheless, the regulatory mechanism between lamin and transcriptional factors in human diseases remains to be further studied.

\section{The Activation of Tumor Protein 53}

DCM is characterized by a progressive conduction system disease, arrhythmia, and systolic impairment (Captur et al., 2018). It has been studied that cyclin-dependent kinase inhibitor 2A (CDKN2A), a downstream target of the E2F pathway, is responsible for activation. LMNA D300N, associated with DCM, results from E2F/DNA damage/TP53 activation. This axis can be a potential intervention target for DCM in laminopathies (Augusto et al., 2020). Furthermore, the impaired crosstalk between endothelial cells (ECs) and cardiomyocytes (CMs) can contribute to the pathogenesis of LMNA-related DCM (Sayed et al., 2020). The other sites of cardiac involvement in DCM contain missense lamin A/C mutation (Arg60Gly) (Porcu et al., 2021).

\section{LAMINB1 MUTATION-RELATED DISEASES Lamin B1 Downregulates Lipid Synthesis Genes}

ADLD is a slow-progressing but fatal neurological disorder in 40-50-year-old adults, usually accompanied by symptoms of autonomic nervous dysfunction, followed by ataxia and cognitive impairment and even the loss of myelin sheath in the central nervous system (CNS) (Chrast et al., 2011; Giorgio et al., 2015). Interestingly, ADLD is the only disease associated with the lamin B1 gene (Padiath, 2019). Otherwise, the pathogenesis of ADLD is overexpressed lamin B1 protein levels due to $L M N B 1$ gene replication or upstream deletion (Takamori et al., 2018). In addition, lamin B1 OE targets oligodendrocytes, thus decreasing the production of myelin sheaths in the CNS. Furthermore, Rolyan et al. also discovered that lamin B1 OE mouses exhibit severe demyelination, axon damage, and neuron loss due to the decreased gene expression of lipid synthesis pathways that play an important role in myelin regulation, ultimately depleting myelin-rich lipids (Rolyan et al., 2015). Indeed, the myelin genes required for oligodendrocyte maturation are sensitively influenced by the nuclear membrane (Lin et al., 2011). However, another study indicated that increased lamin B1 alters the chromatin associated with the region of the nuclear layer, therefore affecting the structure of the nuclear membrane and myelin-related genes (Padiath and Fu, 2010).

In conclusion, the expression of lamin B1 exacerbates ADLD. Despite considerable studies that are accessible, more experiments are necessitated about the lipid-related effects of lamin.

\section{LAMINB2 MUTATION-RELATED DISEASES}

\section{Overactivation of the Complement System}

Mutations in the LMNB2 have been associated with APL, also known as BSS syndrome, which usually begins in childhood or adolescence. Fat loss in BSS is typically characterized by gradual symmetrical subcutaneous fat loss, starting in the face and progressing down the upper part of the body. The subcutaneous fat in the lower abdomen and legs is significantly reduced, while fat storage in the gluteal area and lower limbs tends to be retained or increased (Hegele et al., 2006; Oliveira et al., 2016). CORVILLO et al. analyzed clinical, immunological, and histological events in an 11-year-old girl 
with BSS during a 5-year follow-up, and their results suggest that the overactivation of the complement system in adipose tissue may be responsible for fat loss in BSS patients (Corvillo et al., 2020).

\section{THE REGULATORY MECHANISMS ARE STILL UNCLEAR}

WS or atypical WS is a rare autosomal recessive disorder caused by inherited mutations in the WRN gene and LMNA gene, respectively (Rossi et al., 2010; Wang et al., 2018). In atypical WS associated with the R133L mutation of the LMNA gene, the severity of metabolic complications is positively related to the degree of lipodystrophy (Doh et al., 2009). In addition, Garg et al. investigated the body fat distribution pattern and metabolic abnormalities in two patients with atypical WS carrying R133L heterozygous LMNA mutations. Both patients with LMNA mutations had a unique distribution of body fat, with patient 1 having a fat loss limited to the distal portion of the limbs and an increase in fat deposition in the trunk region, whereas patient 2 had a significant decrease in body fat. In summary, patients with atypical Werner syndrome caused by R133L heterozygous LMNA mutations may present with different types of lipodystrophies, which may present with partial or total body fat loss. Partial lipodystrophy can be further divided into two distinct patterns: one involves the entire limb, mainly including familial partial lipodystrophy, the Dunnigan type, and mandibular dysplasia, while the other happens to involve only the distal limb region (Muchir et al., 2000). However, the regulatory mechanism needs to be further discovered.

Emery-Dreifuss muscular dystrophy (EDMD) is a severe muscular disorder characterized by the early contracture of the elbows, slowly progressive muscle weakness, and cardiomyopathy with conduction block (Onishi et al., 2002). Lamin A/C defects occur both in X-EDMD and AD-EDMD (Niebroj-Dobosz et al., 2003). Lamin A is not only required for lamin B receptor (LBR) retention but also for the localization of transcriptional RNA pol II in muscle cells (Reichart et al., 2004).

Limb girdle muscular dystrophy (LGMD), a type of muscular dystrophy (MD), is manifested as the progressive weakness of muscles (Rajoria et al., 2021). Though the regulatory mechanism is still unclear, a survey has reported that the lipid changes of LGMD are similar to FPLD in skeletal muscle metabolism, mainly exhibiting incomplete fatty acid oxidation and upregulated ketogenesis, which may result from a common underlying cause of muscular metabolic disorders (Boschmann et al., 2010). Actually, LGMD1B is same as FPLD and EDMD (Morris, 2001). Besides, LGMD1B and AD-EDMD are allelic disorders (Muchir et al., 2000).

\section{CONCLUSION}

As a component of nucleus, lamin plays an important role in maintaining nuclear shape, mechanical signaling, stabilizing chromatin, regulating gene expression, and promoting cell cycle progression. Here, we amply review the possible mechanisms of innate or acquired lipid abnormalities caused by lamin in the human diseases, mainly including increased prelamin A accumulation, a dynamic recombination of nuclear layer networks, enhanced lipophagy, activated liver growth hormone receptor signals, segregated SREBP1 at the nuclear margin, adipogenesis, lipid synthesis genes, overactivated complement system, and activated TP53. Those are the targets for lipid defects caused by lamin alterations. Nevertheless, the regulatory mechanism of lamin in lipid metabolism and human diseases remains to be further studied. As a result, targeting lamin should be considered for treating human diseases, which may be a promising disease-reversing strategy for patients.

\section{PROSPECTION}

Lipolysis and autophagy are two central catabolic pathways of lipid decomposition (Zechner et al., 2017). Lipolysis depends on the direct activation of lipase related to LDs, such as adipose triglyceride lipase (ATGL), hormone-sensitive lipase (HSL), and monoglyceryl lipase (MGL). In addition, LDs interact with ATGL activators and inhibitors and then provide energy and basic materials for the synthesis of cell membranes and hormones in the body (Onal et al., 2017). Another lysosomal autophagy pathway that plays an important role in lipid degradation is called lipid autophagy, or lipophagy for short. Lipophagy requires cargo identification accomplished by the interaction between the autophagosome membrane with LC3. Subsequently, LDs are selectively delivered to the lysis chamber for degradation by the autophagic protein (Singh and Cuervo, 2012; Wang, 2016; Kloska et al., 2020; Shin, 2020). Thus, the dysregulation of lipophagy can lead to an abnormal deposition of lipids, therefore seriously affecting cell function and dynamic balance, ultimately resulting in cell death and a variety of diseases, including non-alcoholic fatty liver disease, coronary heart disease, and even cancer (Johnson and Stolzing, 2019).

Nuclear autophagy, a new type of selective autophagy, is responsible for selectively removing damaged or unnecessary nuclear substances in cells (Fu et al., 2018). Nuclear autophagy happens in a variety of conditions, including starvation, rapamycin-induced TORC1 inactivation, nuclear vacuolar junction (NVJ) expansion, and nuclear fibrillary lamina defects (Bo Otto and Thumm, 2020). In 2009, it was first reported that $L M N A / C$ is involved in the development of mammalian nuclear autophagy. Additionally, some nuclear components exist in perinuclear autophagosomes and lysosomes (Park et al., 2009). Dou et al. also found a large amount of endogenous LC3 and a small amount of lipidated LC3-II in the nucleus. Lamin B1 interacts with LC3 to induce nuclear autophagy, which may be enhanced by lipidated LC3 (Dou et al., 2015) (Figure 4). Additionally, the NEM1-spo7/Pah1 axis is very important in the lipid synthesis axis. Meanwhile, this axis is also an important factor to induce nuclear autophagy and correct the localization of micronucleus autophagy factor NVJ1 and nuclear autophagy receptor Atg39 (Rahman et al., 2018; Mirheydari et al., 2020). 


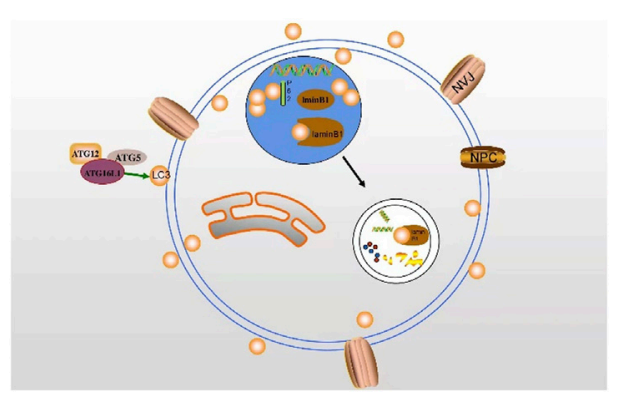

FIGURE 4 | The interaction between LC3 and lamin B1 may induce nuclear autophagy. LC3 transports autophagy membrane and substrate via binding to lamin B1 in the nucleus.

Combined with the above literature, it is not difficult to find that nuclear autophagy is significantly correlated with lipid metabolism. For instance, lamin participates in the occurrence and development of nuclear autophagy by interacting with LC3, and the latter one is an essential factor in the process of lipophagy. As a result, the nuclear autophagy and lipophagy involved in lamin may be not independent of each other, although the specific correlation mechanism and the relationship between lamindependent nuclear autophagy and lipid metabolism deserve to be further studied.

Apart from autophagy, apoptosis is another form of cell death. Apoptosis is characterized by caspase activation, DNA cleavage, and membrane surface modifications that enable apoptotic and phagocytic cells to be recognized, as well as morphological changes such as chromatin concentration and nuclear fragmentation (Jung et al., 2020a). Importantly, lamin also seems to act in the relationship between apoptosis and lipid metabolism. In nonadipose tissues such as liver, heart, kidney, muscle, and islet, the harmful effects caused by excessive accumulation of fat on these organs or systems are called lipotoxicity, which can induce programmed cell death, and lipid apoptosis is the main cellular consequence of lipotoxicity (Schaffer, 2016; Zhang et al., 2019). In all, the involvement of lamins in nuclear autophagy and apoptosis is also a promising point for lipodystrophy and deserves to be further explored in the future.

Chromatin concentration is a nuclear modification characteristic of the active apoptotic phase that follows DNA cleavage and the hydrolysis of certain nuclear proteins by proteases in the caspase family. The cysteine aspartate protease family (caspase) is the main factor affecting protein hydrolysis in the process of apoptosis. The apoptotic caspase can be divided into two types: the initiation of caspase, including caspase- $8,-9$, and -10 , and the execution of caspase, including caspase- $3,-6$, and -7. Caspase- 3 and caspase- 6 are responsible for the cleavage of nuclear proteins PARP and lamin, respectively. Among many apoptosis-related proteins, the hydrolysis of PARP by caspase- 3 is considered as an early indicator of apoptosis. The early cleavage and rapid processing of lamin B by caspase- 6 are regarded as a marker of apoptosis (Villa et al., 1997; Buendia et al., 1999; Eron et al., 2017; Jung et al., 2020b). Meanwhile, lamin A can also be cleaved as a substrate of caspase- 6 . Studies have shown that caspase- 3 cleaves caspase- 6 first in normal cell apoptosis, and caspase- 6 cleaves lamin A/C before apoptosis (Capo-Chichi et al., 2018). Only when lamin A/C is cleaved by caspase- 6 can chromosomal DNA fully coagulate during apoptosis (Yan et al., 2021) (Figure 5). Lamin plays a key role in apoptosis, while the specific mechanism of the involvement of lamin in lipid apoptosis remains to be further studied. For instance, if adipocytes show apoptosis in lipodystrophy, then silencing caspase may prevent its interference with lamin expression, subsequently ameliorating lipid defects.

In $\mathrm{MAD}$, we review the interaction between prelamin $\mathrm{A}$ and SREBP1, suggesting that prelamin A sequestrates SREBP1 at the nuclear border and restricts the translocation of some transcription factors into the nucleus, thereby reducing the pool of normally activated active SREBP1 and leading to the dysdifferentiation of adipocytes (Capanni et al., 2005). Meanwhile, it has been reported that SREBP1c (one of the subtypes of SREBP1) modified by SUMO1 (small ubiquitinlike modifier, also named SUMOylation, is a crucial posttranslational modification that exhibits a strong effect on DNA repair, transcriptional regulation, protein stability, and cell cycle progression) can repress the transcriptional activity of SREBP1c and inhibits lipid production (Pichler et al., 2017; Zeng et al., 2020). Similarly, several lamin A domains can also be modified by SUMOylation. Two typical mutations cause lipid dystrophy (LMNA P.g465d and P.K486N), while only the atypical FPLD2-related p. r482W mutation shows a decrease in lamin A sumoylation. This may provide an alternative mechanism for these atypical lipodystrophies (Simon et al., 2013).

The distribution of adipose tissue is not entirely alike in various types of lipodystrophy. Moreover, the special distribution is an ongoing research. Additionally, the major

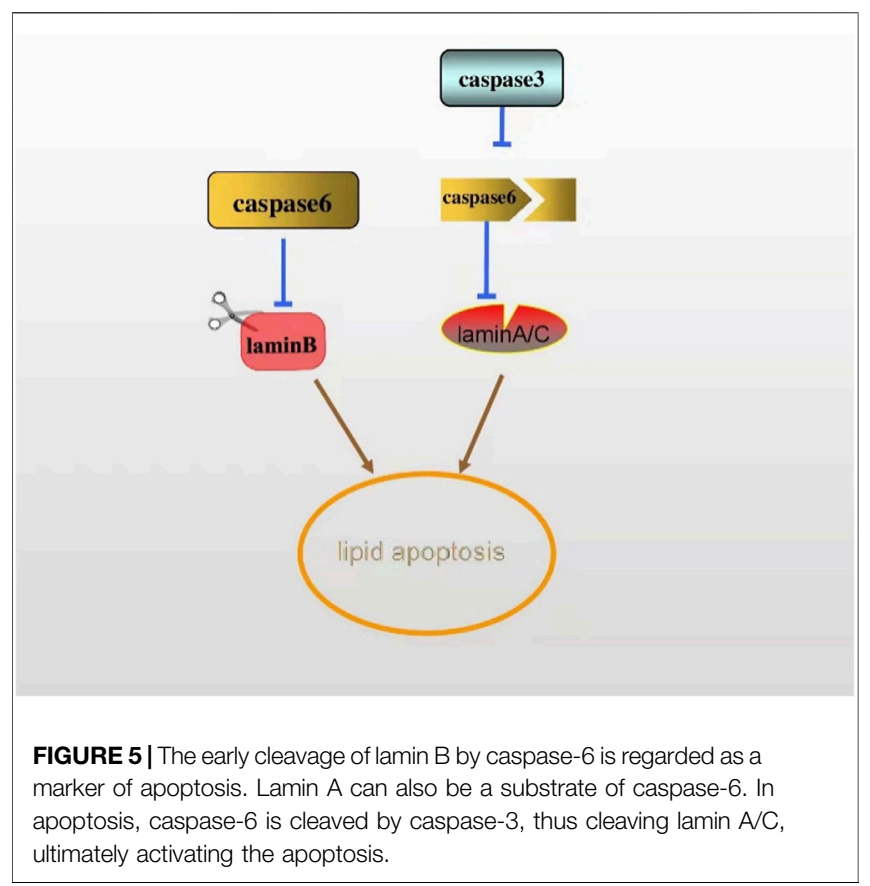


foregone mutated genes in lipid disorders encode proteins forming lipid droplets (Meehan et al., 2016). However, not only the connections between genetic mutation and fat loss but also the cure strategies still need to be discovered. Metreleptin, a recombinant analog of human leptin, is the only drug approved for the treatment of metabolic complications associated with lipodystrophy. This compound is used for the replacement of lipodystrophy accompanied by leptin deficiency without HIV infection (Chevalier et al., 2021). Nonetheless, except for Japan, no country authorizes metreleptin as a drug for lipid issues. Worse, it is not entirely clear whether metreleptin is a benefit or not to lipodystrophy syndrome with normal leptin levels (Oral et al., 2019). Worse still, various complications in the treatment of lipodystrophy have shown to us all. Notably, the most common of which includes weight loss, abdominal pain, hypoglycemia, fatigue, headache, a loss of appetite, injection site reactions (bruising and hives), anti-leptin antibodies, T-cell lymphoma, and infection (Tchang et al., 2015). Even so, metreleptin treatment powerfully alleviates metabolic abnormalities such as hyperglycemia, hypertriglyceridemia, increased hepatic fat content, and elevated liver enzymes alanine transaminase and aspartate transaminase and corrects the hyperphagia of leptin deficiency in patients with generalized lipodystrophy (Akinci and Akinci, 2015).

\section{REFERENCES}

Adam, S. A., Butin-Israeli, V., Cleland, M. M., Shimi, T., and Goldman, R. D. (2013). Disruption of Lamin B1 and Lamin B2 Processing and Localization by Farnesyltransferase Inhibitors. Nucleus 4 (2), 142-150. doi:10.4161/nucl.24089

Afonso, P., Auclair, M., Boccara, F., Vantyghem, M. C., Katlama, C., Capeau, J., et al. (2016). LMNA Mutations Resulting in Lipodystrophy and HIV Protease Inhibitors Trigger Vascular Smooth Muscle Cell Senescence and Calcification: Role of ZMPSTE24 Downregulation. Atherosclerosis 245, 200-211. doi:10.1016/ j.atherosclerosis.2015.12.012

Akinci, G., and Akinci, B. (2015). Metreleptin Treatment in Patients with NonHIV Associated Lipodystrophy. Recent Pat Endocr. Metab. Immune Drug Discov. 9 (2), 74-78. doi:10.2174/1574892811666151111142554

Alfaro, J. F., Gong, C. X., Monroe, M. E., Aldrich, J. T., Clauss, T. R., Purvine, S. O., et al. (2012). Tandem Mass Spectrometry Identifies many Mouse Brain O-GlcNAcylated Proteins Including EGF Domain-specific O-GlcNAc Transferase Targets. Proc. Natl. Acad. Sci. U S A. 109 (19), 7280-7285. doi:10.1073/pnas.1200425109

Augusto, J. B., Eiros, R., Nakou, E., Moura-Ferreira, S., Treibel, T. A., Captur, G., et al. (2020). Dilated Cardiomyopathy and Arrhythmogenic Left Ventricular Cardiomyopathy: a Comprehensive Genotype-Imaging Phenotype Study. Eur. Heart J. Cardiovasc. Imaging 21 (3), 326-336. doi:10.1093/ehjci/jez188

Bagias, C., Xiarchou, A., Bargiota, A., and Tigas, S. (2020). Familial Partial Lipodystrophy (FPLD): Recent Insights. Diabetes Metab. Syndr. Obes. 13, 1531-1544. doi:10.2147/DMSO.S206053

Bidault, G., Garcia, M., Vantyghem, M. C., Ducluzeau, P. H., Morichon, R., Thiyagarajah, K., et al. (2013). Lipodystrophy-linked LMNA p.R482W Mutation Induces Clinical Early Atherosclerosis and In Vitro Endothelial Dysfunction. Arterioscler Thromb. Vasc. Biol. 33 (9), 2162-2171. doi:10. 1161/ATVBAHA.113.301933

Bo Otto, F., and Thumm, M. (2020). Nucleophagy-Implications for Microautophagy and Health. Int. J. Mol. Sci. 21 (12). doi:10.3390/ijms21124506

Boschmann, M., Engeli, S., Moro, C., Luedtke, A., Adams, F., Gorzelniak, K., et al. (2010). LMNA Mutations, Skeletal Muscle Lipid Metabolism, and Insulin
Considering that there is no efficient and safe drug for curing lipodystrophy, targeting relevant mechanisms regulated by lamina may be a promising strategy. The perturbations in mutated lamin are closely associated with the lipid issues, and an understanding of the contribution and influence of lamins in human diseases poses an exciting area for scientific discovery.

\section{AUTHOR CONTRIBUTIONS}

YP wrote the manuscript and QT searched for references. FX and NF was responsible for revising the manuscript.

\section{FUNDING}

This work was jointly supported by the Natural Science Foundation of Hunan Province (2021JJ30628), Clinical medical technology innovation guidance project of Hunan Province (2020SK51904), Natural Science Foundation of Hunan Province (grant number 2019JJ50556), Postgraduate Scientific Research Innovation Project of Hunan Province (CX20210979), Scientific research project of University of South China (Documents of the university of south China (2019)02), the Innovative Province Construction Special Project of Hunan Province (2021SK4031).

Resistance. J. Clin. Endocrinol. Metab. 95 (4), 1634-1643. doi:10.1210/jc. 2009-1293

Buendia, B., Santa-Maria, A., and Courvalin, J. C. (1999). Caspase-dependent Proteolysis of Integral and Peripheral Proteins of Nuclear Membranes and Nuclear Pore Complex Proteins during Apoptosis. J. Cel Sci 112 (Pt 11), 1743-1753. doi:10.1242/jcs.112.11.1743

Capanni, C., Mattioli, E., Columbaro, M., Lucarelli, E., Parnaik, V. K., Novelli, G., et al. (2005). Altered Pre-lamin A Processing Is a Common Mechanism Leading to Lipodystrophy. Hum. Mol. Genet. 14 (11), 1489-1502. doi:10.1093/hmg/ ddi 158

Capo-Chichi, C. D., Cai, K. Q., and Xu, X. X. (2018). Overexpression and Cytoplasmic Localization of Caspase-6 Is Associated with Lamin A Degradation in Set of Ovarian Cancers. Biomark Res. 6, 30. doi:10.1186/ s40364-018-0144-9

Captur, G., Arbustini, E., Bonne, G., Syrris, P., Mills, K., Wahbi, K., et al. (2018). Lamin and the Heart. Heart 104 (6), 468-479. doi:10.1136/heartjnl-2017312338

Cenni, V., D’Apice, M. R., Garagnani, P., Columbaro, M., Novelli, G., Franceschi, C., et al. (2018). Mandibuloacral Dysplasia: A Premature Ageing Disease with Aspects of Physiological Ageing. Ageing Res. Rev. 42, 1-13. doi:10.1016/j.arr. 2017.12.001

Chevalier, B., Lemaitre, M., Leguier, L., Mapihan, K. L., Douillard, C., Jannin, A., et al. (2021). Metreleptin Treatment of Non-HIV Lipodystrophy Syndromes. Presse Med. 50 (3), 104070. doi:10.1016/j.lpm.2021.104070

Chrast, R., Saher, G., Nave, K. A., and Verheijen, M. H. (2011). Lipid Metabolism in Myelinating Glial Cells: Lessons from Human Inherited Disorders and Mouse Models. J. Lipid Res. 52 (3), 419-434. doi:10.1194/jlr.R009761

Corvillo, F., Nozal, P., López-Lera, A., De Miguel, M. P., Piñero-Fernández, J. A., De Lucas, R., et al. (2020). Evidence of Ongoing Complement Activation on Adipose Tissue from an 11-Year-Old Girl with Barraquer-Simons Syndrome. J. Dermatol. 47 (12), 1439-1444. doi:10.1111/1346-8138.15570

de Leeuw, R., Gruenbaum, Y., and Medalia, O. (2018). Nuclear Lamins: Thin Filaments with Major Functions. Trends Cel Biol 28 (1), 34-45. doi:10.1016/j. tcb.2017.08.004

Dittmer, T. A., and Misteli, T. (2011). The Lamin Protein Family. Genome Biol. 12 (5), 222. doi:10.1186/gb-2011-12-5-222 
Doh, Y. J., Kim, H. K., Jung, E. D., Choi, S. H., Kim, J. G., Kim, B. W., et al. (2009). Novel LMNA Gene Mutation in a Patient with Atypical Werner's Syndrome. Korean J. Intern. Med. 24 (1), 68-72. doi:10.3904/kjim.2009.24.1.68

Dou, Z., Xu, C., Donahue, G., Shimi, T., Pan, J. A., Zhu, J., et al. (2015). Autophagy Mediates Degradation of Nuclear Lamina. Nature 527 (7576), 105-109. doi:10. 1038/nature15548

Dunnigan, M. G., Cochrane, M. A., Kelly, A., and Scott, J. W. (1974). Familial Lipoatrophic Diabetes with Dominant Transmission. A New Syndrome. Q. J. Med. 43 (169), 33-48.

Eron, S. J., Raghupathi, K., and Hardy, J. A. (2017). Dual Site Phosphorylation of Caspase-7 by PAK2 Blocks Apoptotic Activity by Two Distinct Mechanisms. Structure 25 (1), 27-39. doi:10.1016/j.str.2016.11.001

Farnsworth, C. C., Wolda, S. L., Gelb, M. H., and Glomset, J. A. (1989). Human Lamin B Contains a Farnesylated Cysteine Residue. J. Biol. Chem. 264 (34), 20422-20429. doi:10.1016/s0021-9258(19)47079-8

Friesen, M., and Cowan, C. A. (2018). FPLD2 LMNA Mutation R482W Dysregulates iPSC-Derived Adipocyte Function and Lipid Metabolism. Biochem. Biophys. Res. Commun. 495 (1), 254-260. doi:10.1016/j.bbrc.2017.11.008

Fu, N., Yang, X., and Chen, L. (2018). Nucleophagy Plays a Major Role in Human Diseases. Curr. Drug Targets 19 (15), 1767-1773. doi:10.2174/ 1389450119666180518112350

Giorgio, E., Robyr, D., Spielmann, M., Ferrero, E., Di Gregorio, E., Imperiale, D., et al. (2015). A Large Genomic Deletion Leads to Enhancer Adoption by the Lamin B1 Gene: a Second Path to Autosomal Dominant Adult-Onset Demyelinating Leukodystrophy (ADLD). Hum. Mol. Genet. 24 (11), 3143-3154. doi:10.1093/hmg/ddv065

Goldberg, M. W., Huttenlauch, I., Hutchison, C. J., and Stick, R. (2008). Filaments Made from A- and B-type Lamins Differ in Structure and Organization. J. Cel Sci 121 (Pt 2), 215-225. doi:10.1242/jcs.022020

Gonzalo, S., Kreienkamp, R., and Askjaer, P. (2017). Hutchinson-Gilford Progeria Syndrome: A Premature Aging Disease Caused by LMNA Gene Mutations. Ageing Res. Rev. 33, 18-29. doi:10.1016/j.arr.2016.06.007

Gruenbaum, Y., and Medalia, O. (2015). Lamins: the Structure and Protein Complexes. Curr. Opin. Cel Biol 32, 7-12. doi:10.1016/j.ceb.2014.09.009

Guénantin, A. C., Briand, N., Bidault, G., Afonso, P., Béréziat, V., Vatier, C., et al. (2014). Nuclear Envelope-Related Lipodystrophies. Semin. Cel Dev Biol 29, 148-157. doi:10.1016/j.semcdb.2013.12.015

Hafidi, M. E., Buelna-Chontal, M., Sánchez-Muñoz, F., and Carbó, R. (2019). Adipogenesis: A Necessary but Harmful Strategy. Int. J. Mol. Sci. 20 (15). doi:10. 3390/ijms20153657

Hegele, R. A., Cao, H., Huff, M. W., and Anderson, C. M. (2000). LMNA R482Q Mutation in Partial Lipodystrophy Associated with Reduced Plasma Leptin Concentration. J. Clin. Endocrinol. Metab. 85 (9), 3089-3093. doi:10.1210/jcem. 85.9.6768

Hegele, R. A., Cao, H., Liu, D. M., Costain, G. A., Charlton-Menys, V., Rodger, N. W., et al. (2006). Sequencing of the Reannotated LMNB2 Gene Reveals Novel Mutations in Patients with Acquired Partial Lipodystrophy. Am. J. Hum. Genet. 79 (2), 383-389. doi:10.1086/505885

Jéru, I., Vatier, C., Araujo-Vilar, D., Vigouroux, C., and Lascols, O. (2017). Clinical Utility Gene Card for: Familial Partial Lipodystrophy. Eur. J. Hum. Genet. 25 (2). doi:10.1038/ejhg.2016.102

Johnson, A. A., and Stolzing, A. (2019). The Role of Lipid Metabolism in Aging, Lifespan Regulation, and Age-Related Disease. Aging Cell 18 (6), e13048. doi:10. 1111/acel.13048

Jung, H. J., Nobumori, C., Goulbourne, C. N., Tu, Y., Lee, J. M., Tatar, A., et al. (2013). Farnesylation of Lamin B1 Is Important for Retention of Nuclear Chromatin during Neuronal Migration. Proc. Natl. Acad. Sci. U S A. 110 (21), E1923-E1932. doi:10.1073/pnas.1303916110

Jung, J. H., Lee, H. J., Kim, J. H., Sim, D. Y., Im, E., Kim, S., et al. (2020). Colocalization of MID1IP1 and C-Myc Is Critically Involved in Liver Cancer Growth via Regulation of Ribosomal Protein L5 and L11 and CNOT2. Cells 9 (4), 985. doi:10.3390/cells9040985

Jung, S., Jeong, H., and Yu, S. W. (2020). Autophagy as a Decisive Process for Cell Death. Exp. Mol. Med. 52 (6), 921-930. doi:10.1038/s12276-020-0455-4

Kim, Y., Bayona, P. W., Kim, M., Chang, J., Hong, S., Park, Y., et al. (2018). Macrophage Lamin A/C Regulates Inflammation and the Development of Obesity-Induced Insulin Resistance. Front. Immunol. 9, 696. doi:10.3389/ fimmu.2018.00696
Kloska, A., Węsierska, M., Malinowska, M., Gabig-Cimińska, M., and Jakóbkiewicz-Banecka, J. (2020). Lipophagy and Lipolysis Status in Lipid Storage and Lipid Metabolism Diseases. Int. J. Mol. Sci. 21 (17), 6113. doi:10.3390/ijms21176113

Köbberling, J., Willms, B., Kattermann, R., and Creutzfeldt, W. (1975). Lipodystrophy of the Extremities. A Dominantly Inherited Syndrome Associated with Lipatrophic Diabetes. Humangenetik 29 (2), 111-120. doi:10.1007/BF00430347

Krawiec, P., Mełges, B., Pac-Kożuchowska, E., Mroczkowska-Juchkiewicz, A., and Czerska, K. (2016). Fitting the Pieces of the Puzzle Together: a Case Report of the Dunnigan-type of Familial Partial Lipodystrophy in the Adolescent Girl. BMC Pediatr. 16, 38. doi:10.1186/s12887-016-0581-2

Kuchay, S., Wang, H., Marzio, A., Jain, K., Homer, H., Fehrenbacher, N., et al. (2019). GGTase3 Is a Newly Identified Geranylgeranyltransferase Targeting a Ubiquitin Ligase. Nat. Struct. Mol. Biol. 26 (7), 628-636. doi:10.1038/s41594019-0249-3

Kwan, R., Brady, G. F., Brzozowski, M., Weerasinghe, S. V., Martin, H., Park, M. J., et al. (2017). Hepatocyte-Specific Deletion of Mouse Lamin A/C Leads to MaleSelective Steatohepatitis. Cell Mol Gastroenterol Hepatol 4 (3), 365-383. doi:10. 1016/j.jcmgh.2017.06.005

Laver, T. W., Patel, K. A., Colclough, K., Curran, J., Dale, J., Davis, N., et al. (2018). PLIN1 Haploinsufficiency Is Not Associated with Lipodystrophy. J. Clin. Endocrinol. Metab. 103 (9), 3225-3230. doi:10.1210/jc.2017-02662

Li, X., Yang, M., Li, Z., Xue, M., Shangguan, Z., Ou, Z., et al. (2016). Fructus Xanthii Improves Lipid Homeostasis in the Epididymal Adipose Tissue of Rats Fed a High-Fat Diet. Mol. Med. Rep. 13 (1), 787-795. doi:10.3892/mmr. 2015.4628

Lin, S. T., Ptácek, L. J., and Fu, Y. H. (2011). Adult-onset Autosomal Dominant Leukodystrophy: Linking Nuclear Envelope to Myelin. J. Neurosci. 31 (4), 1163-1166. doi:10.1523/JNEUROSCI.5994-10.2011

Machowska, M., Piekarowicz, K., and Rzepecki, R. (2015). Regulation of Lamin Properties and Functions: Does Phosphorylation Do it All?. Open Biol. 5 (11). doi:10.1098/rsob.150094

Mahdi, L., Kahn, A., Dhamija, R., and Vargas, H. E. (2020). Hepatic Steatosis Resulting from LMNA-Associated Familial Lipodystrophy. ACG Case Rep. J. 7 (4), e00375. doi:10.14309/cri.0000000000000375

Mantovani, A., and Dalbeni, A. (2020). NAFLD, MAFLD and DAFLD. Dig. Liver Dis. 52 (12), 1519-1520. doi:10.1016/j.dld.2020.09.013

Maraldi, N. M., Capanni, C., Cenni, V., Fini, M., and Lattanzi, G. (2011). Laminopathies and Lamin-Associated Signaling Pathways. J. Cel Biochem 112 (4), 979-992. doi:10.1002/jcb.22992

Meehan, C. A., Cochran, E., Kassai, A., Brown, R. J., and Gorden, P. (2016). Metreleptin for Injection to Treat the Complications of Leptin Deficiency in Patients with Congenital or Acquired Generalized Lipodystrophy. Expert Rev. Clin. Pharmacol. 9 (1), 59-68. doi:10.1586/17512433.2016.1096772

Mirheydari, M., Dey, P., Stukey, G. J., Park, Y., Han, G. S., and Carman, G. M. (2020). The Spo7 Sequence LLI Is Required for Nem1-Spo7/Pah1 Phosphatase cascade Function in Yeast Lipid Metabolism. J. Biol. Chem. 295 (33), 11473-11485. doi:10.1074/jbc.RA120.014129

Moriuchi, T., Kuroda, M., Kusumoto, F., Osumi, T., and Hirose, F. (2016). Lamin A Reassembly at the End of Mitosis Is Regulated by its SUMO-Interacting Motif. Exp. Cel Res 342 (1), 83-94. doi:10.1016/j.yexcr.2016.02.016

Morris, G. E. (2001). The Role of the Nuclear Envelope in Emery-Dreifuss Muscular Dystrophy. Trends Mol. Med. 7 (12), 572-577. doi:10.1016/s14714914(01)02128-1

Muchir, A., Bonne, G., van der Kooi, A. J., van Meegen, M., Baas, F., Bolhuis, P. A., et al. (2000). Identification of Mutations in the Gene Encoding Lamins A/C in Autosomal Dominant Limb Girdle Muscular Dystrophy with Atrioventricular Conduction Disturbances (LGMD1B). Hum. Mol. Genet. 9 (9), 1453-1459. doi:10.1093/hmg/9.9.1453

Najdi, F., Krüger, P., and Djabali, K. (2021). Impact of Progerin Expression on Adipogenesis in Hutchinson-Gilford Progeria Skin-Derived Precursor Cells. Cells 10 (7). doi:10.3390/cells10071598

Niebroj-Dobosz, I., Fidzianska, A., and Hausmanowa-Petrusewicz, I. (2003). Expression of Emerin and Lamins in Muscle of Patients with Different Forms of Emery-Dreifuss Muscular Dystrophy. Acta Myol 22 (2), 52-57.

Novelli, G., Muchir, A., Sangiuolo, F., Helbling-Leclerc, A., D'Apice, M. R., Massart, C., et al. (2002). Mandibuloacral Dysplasia Is Caused by a 
Mutation in LMNA-Encoding Lamin A/C. Am. J. Hum. Genet. 71 (2), 426-431. doi: $10.1086 / 341908$

Oliveira, J., Freitas, P., Lau, E., and Carvalho, D. (2016). Barraquer-Simons Syndrome: a Rare Form of Acquired Lipodystrophy. BMC Res. Notes 9, 175. doi:10.1186/s13104-016-1975-9

Onal, G., Kutlu, O., Gozuacik, D., and Dokmeci Emre, S. (2017). Lipid Droplets in Health and Disease. Lipids Health Dis. 16 (1), 128. doi:10.1186/s12944-0170521-7

Onishi, Y., Higuchi, J., Ogawa, T., Namekawa, A., Hayashi, H., Odakura, H., et al. (2002). The First Japanese Case of Autosomal Dominant Emery-Dreifuss Muscular Dystrophy with a Novel Mutation in the Lamin A/C Gene. Rinsho Shinkeigaku 42 (2), 140-144.

Oral, E. A., Gorden, P., Cochran, E., Araújo-Vilar, D., Savage, D. B., Long, A., et al. (2019). Long-term Effectiveness and Safety of Metreleptin in the Treatment of Patients with Partial Lipodystrophy. Endocrine 64 (3), 500-511. doi:10.1007/ s12020-019-01862-8

Östlund, C., Hernandez-Ono, A., and Shin, J. Y. (2020). The Nuclear Envelope in Lipid Metabolism and Pathogenesis of NAFLD. Biology (Basel) 9 (10), 338. doi:10.3390/biology9100338

Özen, S., Akıncı, B., and Oral, E. A. (2020). Current Diagnosis, Treatment and Clinical Challenges in the Management of Lipodystrophy Syndromes in Children and Young People. J. Clin. Res. Pediatr. Endocrinol. 12 (1), 17-28. doi:10.4274/jcrpe.galenos.2019.2019.0124

Padiath, Q. S. (2019). Autosomal Dominant Leukodystrophy: A Disease of the Nuclear Lamina. Front Cel Dev Biol 7, 41. doi:10.3389/fcell.2019.00041

Padiath, Q. S., and Fu, Y. H. (2010). Autosomal Dominant Leukodystrophy Caused by Lamin B1 Duplications a Clinical and Molecular Case Study of Altered Nuclear Function and Disease. Methods Cel Biol 98, 337-357. doi:10.1016/ S0091-679X(10)98014-X

Park, Y. E., Hayashi, Y. K., Bonne, G., Arimura, T., Noguchi, S., Nonaka, I., et al. (2009). Autophagic Degradation of Nuclear Components in Mammalian Cells. Autophagy 5 (6), 795-804. doi:10.4161/auto.8901

Pichler, A., Fatouros, C., Lee, H., and Eisenhardt, N. (2017). SUMO Conjugation - a Mechanistic View. Biomol. Concepts 8 (1), 13-36. doi:10.1515/bmc-2016-0030

Porcu, M., Corda, M., Pasqualucci, D., Binaghi, G., Sanna, N., Matta, G., et al. (2021). A Very Long-Term Observation of a Family with Dilated Cardiomyopathy and Overlapping Phenotype from Lamin A/C Mutation. J. Cardiovasc. Med. (Hagerstown) 22 (1), 53-58. doi:10.2459/JCM. 0000000000001060

Povlsen, L. K., Beli, P., Wagner, S. A., Poulsen, S. L., Sylvestersen, K. B., Poulsen, J. W., et al. (2012). Systems-wide Analysis of Ubiquitylation Dynamics Reveals a Key Role for PAF15 Ubiquitylation in DNA-Damage Bypass. Nat. Cel Biol 14 (10), 1089-1098. doi:10.1038/ncb2579

Rahman, M. A., Mostofa, M. G., and Ushimaru, T. (2018). The Nem1/Spo7-Pah1/ lipin axis Is Required for Autophagy Induction after TORC1 Inactivation. FEBS J. 285 (10), 1840-1860. doi:10.1111/febs.14448

Rajoria, K., Singh, S. K., and Dadhich, S. (2021). Ayurvedic Management in Limb Girdle Muscular Dystrophy - A Case Report. J. Ayurveda Integr. Med. 13, 100486. doi:10.1016/j.jaim.2021.07.002

Rao, R. A., Ketkar, A. A., Kedia, N., Krishnamoorthy, V. K., Lakshmanan, V., Kumar, P., et al. (2019). KMT1 Family Methyltransferases Regulate Heterochromatin-Nuclear Periphery Tethering via Histone and Nonhistone Protein Methylation. EMBO Rep. 20 (5), e43260. doi:10.15252/ embr.201643260

Reichart, B., Klafke, R., Dreger, C., Krüger, E., Motsch, I., Ewald, A., et al. (2004). Expression and Localization of Nuclear Proteins in Autosomal-Dominant Emery-Dreifuss Muscular Dystrophy with LMNA R377H Mutation. BMC Cel Biol 5, 12. doi:10.1186/1471-2121-5-12

Rolyan, H., Tyurina, Y. Y., Hernandez, M., Amoscato, A. A., Sparvero, L. J., Nmezi, B. C., et al. (2015). Defects of Lipid Synthesis Are Linked to the Age-dependent Demyelination Caused by Lamin B1 Overexpression. J. Neurosci. 35 (34), 12002-12017. doi:10.1523/JNEUROSCI.1668-15.2015

Rossi, M. L., Ghosh, A. K., and Bohr, V. A. (2010). Roles of Werner Syndrome Protein in protection of Genome Integrity. DNA Repair (Amst) 9 (3), 331-344. doi:10.1016/j.dnarep.2009.12.011

Ruiz de Eguino, G., Infante, A., Schlangen, K., Aransay, A. M., Fullaondo, A., Soriano, M., et al. (2012). Spl Transcription Factor Interaction with Accumulated Prelamin a Impairs Adipose Lineage Differentiation in
Human Mesenchymal Stem Cells: Essential Role of Sp1 in the Integrity of Lipid Vesicles. Stem Cell Transl Med 1 (4), 309-321. doi:10.5966/sctm.20110010

Sayed, N., Liu, C., Ameen, M., Himmati, F., Zhang, J. Z., Khanamiri, S., et al. (2020). Clinical Trial in a Dish Using iPSCs Shows Lovastatin Improves Endothelial Dysfunction and Cellular Cross-Talk in LMNA Cardiomyopathy. Sci. Transl Med. 12 (554), eaax9276. doi:10.1126/scitranslmed.aax9276

Schaffer, J. E. (2016). Lipotoxicity: Many Roads to Cell Dysfunction and Cell Death: Introduction to a Thematic Review Series. J. Lipid Res. 57 (8), 1327-1328. doi:10.1194/jlr.E069880

Shin, D. W. (2020). Lipophagy: Molecular Mechanisms and Implications in Metabolic Disorders. Mol. Cell 43 (8), 686-693. doi:10.14348/molcells.2020. 0046

Simon, D. N., Domaradzki, T., Hofmann, W. A., and Wilson, K. L. (2013). Lamin A Tail Modification by SUMO1 Is Disrupted by Familial Partial LipodystrophyCausing Mutations. Mol. Biol. Cel 24 (3), 342-350. doi:10.1091/mbc.E12-070527

Simon, D. N., Wriston, A., Fan, Q., Shabanowitz, J., Florwick, A., Dharmaraj, T., et al. (2018). OGT (O-GlcNAc Transferase) Selectively Modifies Multiple Residues Unique to Lamin A. Cells 7 (5). doi:10.3390/cells7050044

Singh, R., and Cuervo, A. M. (2012). Lipophagy: Connecting Autophagy and Lipid Metabolism. Int. J. Cel Biol 2012, 282041. doi:10.1155/2012/282041

Snider, N. T., and Omary, M. B. (2014). Post-translational Modifications of Intermediate Filament Proteins: Mechanisms and Functions. Nat. Rev. Mol. Cel Biol 15 (3), 163-177. doi:10.1038/nrm3753

Stiekema, M., van Zandvoort, M. A. M. J., Ramaekers, F. C. S., and Broers, J. L. V. (2020). Structural and Mechanical Aberrations of the Nuclear Lamina in Disease. Cells 9 (8). doi:10.3390/cells9081884

Takamori, Y., Hirahara, Y., Wakabayashi, T., Mori, T., Koike, T., Kataoka, Y., et al. (2018). Differential Expression of Nuclear Lamin Subtypes in the Neural Cells of the Adult Rat Cerebral Cortex. IBRO Rep. 5, 99-109. doi:10.1016/j.ibror. 2018.11.001

Tchang, B. G., Shukla, A. P., and Aronne, L. J. (2015). Metreleptin and Generalized Lipodystrophy and Evolving Therapeutic Perspectives. Expert Opin. Biol. Ther. 15 (7), 1061-1075. doi:10.1517/14712598.2015.1052789

Tu, Y., Sánchez-Iglesias, S., Araújo-Vilar, D., Fong, L. G., and Young, S. G. (2016). LMNA Missense Mutations Causing Familial Partial Lipodystrophy Do Not lead to an Accumulation of Prelamin A. Nucleus 7 (5), 512-521. doi:10.1080/ 19491034.2016.1242542

Verstraeten, V. L., Renes, J., Ramaekers, F. C., Kamps, M., Kuijpers, H. J., Verheyen, F., et al. (2011). Reorganization of the Nuclear Lamina and Cytoskeleton in Adipogenesis. Histochem. Cel Biol 135 (3), 251-261. doi:10.1007/s00418-0110792-4

Villa, P., Kaufmann, S. H., and Earnshaw, W. C. (1997). Caspases and Caspase Inhibitors. Trends Biochem. Sci. 22 (10), 388-393. doi:10.1016/s0968-0004(97) 01107-9

Wagner, S. A., Beli, P., Weinert, B. T., Nielsen, M. L., Cox, J., Mann, M., et al. (2011). A Proteome-wide, Quantitative Survey of In Vivo Ubiquitylation Sites Reveals Widespread Regulatory Roles. Mol. Cel Proteomics 10 (10), M111-M013284. doi:10.1074/mcp.M111.013284

Wang, C. W. (2016). Lipid Droplets, Lipophagy, and beyond. Biochim. Biophys. Acta 1861 (8 Pt B), 793-805. doi:10.1016/j.bbalip.2015.12.010

Wang, L. R., Radonjic, A., Dilliott, A. A., McIntyre, A. D., and Hegele, R. A. (2018). A De Novo POLD1 Mutation Associated with Mandibular Hypoplasia, Deafness, Progeroid Features, and Lipodystrophy Syndrome in a Family with Werner Syndrome. J. Investig. Med. High Impact Case Rep. 6, 2324709618786770. doi:10.1177/2324709618786770

Wang, S., Huang, X., Sun, D., Xin, X., Pan, Q., Peng, S., et al. (2012). Extensive Crosstalk between O-GlcNAcylation and Phosphorylation Regulates Akt Signaling. PLoS One 7 (5), e37427. doi:10.1371/journal. pone. 0037427

Weber, K., Plessmann, U., and Traub, P. (1989). Maturation of Nuclear Lamin A Involves a Specific Carboxy-Terminal Trimming, Which Removes the Polyisoprenylation Site from the Precursor; Implications for the Structure of the Nuclear Lamina. FEBS Lett. 257 (2), 411-414. doi:10.1016/0014-5793(89) 81584-4

Weinert, B. T., Schölz, C., Wagner, S. A., Iesmantavicius, V., Su, D., Daniel, J. A., et al. (2013). Lysine Succinylation Is a Frequently Occurring Modification in 
Prokaryotes and Eukaryotes and Extensively Overlaps with Acetylation. Cell Rep 4 (4), 842-851. doi:10.1016/j.celrep.2013.07.024

Winter-Vann, A. M., and Casey, P. J. (2005). Post-prenylation-processing Enzymes as New Targets in Oncogenesis. Nat. Rev. Cancer 5 (5), 405-412. doi:10.1038/ nrc1612

Yan, J., Xie, Y., Si, J., Gan, L., Li, H., Sun, C., et al. (2021). Crosstalk of the Caspase Family and Mammalian Target of Rapamycin Signaling. Ijms 22 (2), 817. doi:10.3390/ijms22020817

Zechner, R., Madeo, F., and Kratky, D. (2017). Cytosolic Lipolysis and Lipophagy: Two Sides of the Same coin. Nat. Rev. Mol. Cel Biol 18 (11), 671-684. doi:10. 1038/nrm.2017.76

Zeng, M., Liu, W., Hu, Y., and Fu, N. (2020). Sumoylation in Liver Disease. Clin. Chim. Acta 510, 347-353. doi:10.1016/j.cca.2020.07.044

Zhang, E., Lu, X., Yin, S., Yan, M., Lu, S., Fan, L., et al. (2019). The Functional Role of Bax/Bak in Palmitate-Induced Lipoapoptosis. Food Chem. Toxicol. 123, 268-274. doi:10.1016/j.fct.2018.11.011
Conflict of Interest: The authors declare that the research was conducted in the absence of any commercial or financial relationships that could be construed as a potential conflict of interest.

Publisher's Note: All claims expressed in this article are solely those of the authors and do not necessarily represent those of their affiliated organizations, or those of the publisher, the editors and the reviewers. Any product that may be evaluated in this article, or claim that may be made by its manufacturer, is not guaranteed or endorsed by the publisher.

Copyright $\odot 2022$ Peng, Tang, Xiao and Fu. This is an open-access article distributed under the terms of the Creative Commons Attribution License (CC BY). The use, distribution or reproduction in other forums is permitted, provided the original author(s) and the copyright owner(s) are credited and that the original publication in this journal is cited, in accordance with accepted academic practice. No use, distribution or reproduction is permitted which does not comply with these terms. 


\section{GLOSSARY}

ADLD autosomal dominant leukodystrophy

AD-EDMD autosomal dominant form of Emery-Dreifuss muscular dystrophy

APL acquired partial lipodystrophy

ATGL adipose triglyceride lipase

BSS Barraquer-Simons syndrome

CDKN2A cyclin-dependent kinase inhibitor 2A

CNS central nervous system

CMs cardiomyocytes

DM diabetes mellitus

DCM dilated cardiomyopathy

ECs endothelial cells

EDMD Emery-Dreifuss muscular dystrophy

ERK extracellular regulated protein kinase

GH growth hormone

HSL hormone-sensitive lipase

hMSC human mesenchymal stem cell

HGPS Hutchinson-Gilford progeria syndrome

IFS intermediate filaggrin

INM inner nuclear membrane

JAK2 Janus kinase 2
LGMD limb girdle muscular dystrophy

LGMD1B limb girdle muscular dystrophy type $1 \mathrm{~b}$

LD lipid droplet

LBR lamin B receptor

MADA type A mandibuloacral dysplasia

MADB type B mandibuloacral dysplasia

MAD mandibular sacral dysplasia

MAFLD metabolic associated fatty liver disease

MD muscular dystrophy

MGL monoglyceryl lipase

NLS nuclear localization signal

NVJ nuclear vacuolar junction

ONM outer nuclear membrane

OE overexpression

PPAR gamma peroxisome proliferator activated receptor gamma

PI protease inhibitor

PTMs post-translational modification

SUMOylation small ubiquitin-like modifier

Stat5 transcription activator

SREBP1 cholesterol regulatory element binding protein 1

WS Werner syndrome

ZMPSTE24 zinc metalloproteinase 